\title{
Association of Coffee Consumption with Sarcopenia in Korean Elderly Men: Analysis Using the Korea National Health and Nutrition Examination Survey, 2008-2011
}

\author{
Hyewon Chung ${ }^{1,2}$, Ji Hyun Moon ${ }^{1,2}$, Ju II Kim', Mi Hee Kong ${ }^{1,3}$, Jung Sik Huh ${ }^{4,5}$, Hyeon Ju Kim ${ }^{1,3, *}$ \\ 'Department of Family Medicine, Jeju National University Hospital, Jeju, Korea \\ ${ }^{2}$ Department of Medicine, Graduate School of Jeju National University, Jeju, Korea \\ ${ }^{3}$ Department of Family Medicine, Jeju National University, Jeju, Korea \\ ${ }^{4}$ Department of Urology, Jeju National University, Jeju, Korea \\ ${ }^{5}$ Department of Urology, Jeju National University Hospital, Jeju, Korea
}

Background: Sarcopenia is an age-related loss of muscle mass and strength. Coffee has antioxidant and anti-inflammatory properties that have been shown to be inversely related to the mechanism of sarcopenia. While there have been some studies on the effect of coffee on sarcopenia in animals, studies on the topic in humans are rare. Therefore, we investigated this relationship in elderly Korean men.

Methods: The cross-sectional data were derived from the 2008-2011 Korea National Health and Nutrition Examination Survey. After applying the exclusion criteria, the study sample consisted of 1,781 men who were at least 60 years of age. Study participants were identified as having sarcopenia if their appendicular skeletal muscle mass divided by height squared was less than two standard deviations below the gender-specific mean of this value for young adults. Daily coffee consumption amounts were categorized as $<1$ cup, 1 cup, 2 cups, and $\geq 3$ cups.

Results: Compared to the group of individuals who drank less than one cup of coffee a day, people who consumed at least 3 cups (adjusted odds ratio, 0.43 ; $95 \%$ confidence interval, 0.20 to 0.94 ) showed significantly decreased sarcopenia; however, the decrease was not significant when the daily coffee consumption was 1 or 2 cups. In multivariate logistic regression models, significant associations were observed between sarcopenia and coffee consumption ( $\mathrm{P}$ for trend $=0.039$ ).

Conclusion: The results of this study suggest that consuming at least 3 cups of coffee per day was associated with a lower prevalence of sarcopenia in elderly Korean elderly men.

Keywords: Coffee; Sarcopenia; Aged; Koreans 


\section{INTRODUCTION}

Coffee has phenolic compounds such as chlorogenic acid and caffeic acid that have strong antioxidant activity and anti-inflammatory effects, as well as the potential to induce autophagy. ${ }^{1-4)}$ Considering these pharmacological benefits of coffee, studies have been conducted to investigate the association between coffee consumption and several clinical conditions. The results of these studies suggest that habitual coffee consumption is associated with lower prevalence of diabetes or pre-diabetes, ${ }^{5)}$ insulin resistance, ${ }^{6)}$ stroke, ${ }^{7)}$ Parkinson's disease, ${ }^{8)}$ and cardiovascular disease. ${ }^{9)}$ There is also evidence that coffee consumption is associated with decreased risks for some types of cancer. ${ }^{10)}$

Sarcopenia has been defined as the loss of muscle mass and strength, ${ }^{11)}$ which is progressive, and mainly related to age. ${ }^{12)}$ It can result in mobility disorders, physical disability, poor quality of life, and even death. ${ }^{13)}$ Several factors are related to the pathogenesis of sarcopenia. Age-related changes such as decreased levels and sensitivity of anabolic hormones, lack of physical activity, nutritional deficiencies, and comorbid diseases all contribute to the onset and progression of sarcopenia. These changes are hypothesized to lead inflammation with increased circulating levels of inflammatory cytokines, oxidative stress, suppressed muscle autophagy, and increased apoptosis by altering intra- and extracellular processes. ${ }^{14-16)}$ In addition, oxidative metabolism generates reactive oxygen species, which alters skeletal muscle mitochondrial DNA and can lead to sarcopenia. ${ }^{17)}$

In one animal trial of aged mice to examine the effect of coffee on skeletal muscles, coffee attenuated the reduction of age-related muscle weight and muscle power, and stimulated regeneration of injured muscle compared to controls. Furthermore, pro-inflammatory mediators such as tumor necrosis factor-alpha and interleukin 6 decreased after coffee treatment. ${ }^{18)}$

Despite these findings, studies of the effect of coffee on sarcopenia in humans are uncommon. In this epidemiological study, we analyzed the association of coffee consumption and sarcopenia in elderly Korean men.

\section{METHODS}

\section{Study Population}

Data are from the cross-sectional survey of the second and third year (2008-2009) of the fourth Korea National Health and Nutrition Examination Survey (KNHANES IV) and the first and second year (20102011) of the KNHANES V, conducted by the Korea Centers for Disease Control and Prevention (KCDC). Originally conducted in 1998, the KNHANES is performed periodically as a cross-sectional and nationally representative survey to investigate the general health and nutritional status of the Korean population. It uses a complex, stratified, multistage, probability-cluster sampling method, which enables the collection of extensive and nationally representative data. The protocols for the KNHANES IV and V were approved by the institutional review board of the KCDC (2008-04EXP-01-C, 2009-01CON-03-2C,
2010-02CON-21-C, 2011-02CON-06-C). All participants provided written informed consent before completing the survey, which included a health interview, a nutritional survey, and a health examination. Data were collected via household interviews and standardized physical examinations. During the period from 2008 to 2011, 9,043 males took part in the KNHANES, and 2,451 men aged 60 years or older. Within the sample of men who were at least 60 years of age, individuals with stroke (144), myocardial infarction or angina (153), liver cirrhosis (17), renal failure (12), or cancer (344) were excluded. The final 1,781 subjects were included for the analysis.

\section{Assessment of Coffee Intake}

Self-reported coffee consumption data were obtained through dietary interviews. A food frequency questionnaire was used to assess the usual dietary intake over the preceding year. Participants were asked how many cups of coffee they drank during a day, a week or a month. In the questionnaire, the frequency of coffee intake was classified into ten categories: rarely, 6-11 cups per year, 1 cup per month, 2-3 cups per month, 1 cup per week, 2-3 cups per week, 4-6 cups per week, 1 cup per day, 2 cups per day, and 3 or more cups per day. Four coffee consumption groups were constructed: less than 1 cup per day $(<1$ cup), 1 cup per day ( 1 cup), 2 cups per day ( 2 cups), and 3 or more cups per day ( $\geq 3$ cups), because of the excessively small samples and the small differences between data of each section of the four groups. No distinction was made between caffeinated and decaffeinated coffee, or between the individual types of coffee (boiled, filtered, or instant).

\section{Definition of Sarcopenia}

Whole and regional body compositions of participants were measured using dual-energy X-ray absorptiometry (DXA, Discovery-W fan-beam densitometer; Hologic Inc., Bedford, MA, USA). According to the definition of sarcopenia proposed by Baumgartner et al., ${ }^{11)}$ we summed the muscle mass of the four limbs from DXA as appendicular skeletal muscle mass (ASM) and defined a skeletal muscle mass index (SMI) as ASM divided by height squared, ASM $/$ height $^{2}\left(\mathrm{~kg} / \mathrm{m}^{2}\right)$. The cutoff value for sarcopenia was 2 standard deviations (SDs) below the mean SMI of young males (20-39 years). Participants were divided into groups with and without sarcopenia.

\section{Statistical Analysis}

We applied weights from the KNHANES to calculate estimated means, proportions, and standard errors for the Korean population. We also used complex-sample logistic regression analysis to evaluate the relationship between sarcopenia and coffee consumption. Results were considered to be statistically significant when the P-value was less than 0.05 . To estimate $P$ for trends, coffee consumption group variable was regarded as a continuous variable in the trend analysis. Data were analyzed using IBM SPSS ver. 20.0 for Windows (IBM Corp., Armonk, NY, USA). 


\section{RESULTS}

\section{Comparison of the Groups with and without Sarcopenia}

The clinical characteristics of the Korean elderly men who were included in this analysis are shown in Table 1 . These estimates were calculated using complex-sample general linear regression and analyzed using complex-sample logistic regression methods (Table 1). All 1,781 study subjects were men aged 60 years or older and their mean age was 68.2 years. With the sarcopenia definition as described in the methods, the cutoff point of sarcopenia in men was $6.07 \mathrm{~kg} / \mathrm{m}^{2}$. The mean age of men with sarcopenia was $72.0 \pm 0.68$ years, which was significantly higher than in men without sarcopenia, $67.9 \pm 0.19$ years $(\mathrm{P}<0.001)$. Similarly, the mean height and weight of individuals without sarcopenia was $165.8 \pm 0.17 \mathrm{~cm}$ and $65.5 \pm 0.28 \mathrm{~kg}$, respectively, while those of the sarcopenia group was $163.8 \pm 0.57 \mathrm{~cm}(\mathrm{P}=0.001)$ and $51.1 \pm 0.71 \mathrm{~kg}(\mathrm{P}<0.001)$. Furthermore, the mean SMI in men without sarcopenia was $7.4 \pm 0.03 \mathrm{~kg} / \mathrm{m}^{2}$ and $5.7 \pm 0.04 \mathrm{~kg} / \mathrm{m}^{2}$ in the group with sarcopenia $(\mathrm{P}<0.001)$. Smoking status and exercise frequency were significantly different between two groups $(\mathrm{P}=0.004, \mathrm{P}=0.006$, respectively), but there was no difference in alcohol consumption frequency

Table 1. Clinical characteristics of the participants with and without sarcopenia

\begin{tabular}{|c|c|c|c|}
\hline Characteristic & $\begin{array}{l}\text { Without sarcopenia } \\
\qquad\left(\mathrm{N}=1,656^{\star}\right)\end{array}$ & $\begin{array}{l}\text { With sarcopenia } \\
\quad\left(\mathrm{N}=125^{*}\right)\end{array}$ & $\mathrm{P}$-value \\
\hline Age (y) & $67.9 \pm 0.19$ & $72.0 \pm 0.68$ & $<0.001$ \\
\hline $60-69$ & $64.0(1.3)$ & $38.5(4.5)$ & $<0.001$ \\
\hline $70-79$ & $31.2(1.2)$ & $49.0(5.0)$ & \\
\hline$\geq 80$ & $4.8(0.6)$ & $21.5(3.6)$ & \\
\hline Height (cm) & $165.8 \pm 0.17$ & $163.8 \pm 0.57$ & 0.001 \\
\hline Weight (kg) & $65.5 \pm 0.28$ & $51.1 \pm 0.71$ & $<0.001$ \\
\hline Waist circumference $(\mathrm{cm})$ & $86.3 \pm 0.27$ & $73.9 \pm 0.97$ & $<0.001$ \\
\hline Body mass index $\left(\mathrm{kg} / \mathrm{m}^{2}\right)$ & $23.8 \pm 0.09$ & $19.0 \pm 0.24$ & $<0.001$ \\
\hline ASM $(\mathrm{kg})$ & $20.4 \pm 0.09$ & $15.4 \pm 0.16$ & $<0.001$ \\
\hline ASM/height ${ }^{2}\left(\mathrm{~kg} / \mathrm{m}^{2}\right)$ & $7.4 \pm 0.03$ & $5.7 \pm 0.04$ & $<0.001$ \\
\hline Coffee (cup/d) & & & 0.034 \\
\hline$<1$ & $30.7(1.4)$ & $43.4(5.7)$ & \\
\hline 1 & $27.1(1.4)$ & $28.2(4.5)$ & \\
\hline 2 & $24.4(1.3)$ & $18.8(4.2)$ & \\
\hline$\geq 3$ & $17.9(1.2)$ & $9.6(2.8)$ & \\
\hline Smoking & & & 0.004 \\
\hline Never & $16.2(1.1)$ & $9.1(2.6)$ & \\
\hline Former & $27.8(1.5)$ & $17.2(4.1)$ & \\
\hline Current & $56.0(1.7)$ & $73.7(4.7)$ & \\
\hline Alcohol & & & 0.618 \\
\hline Never & $23.8(1.2)$ & $27.9(4.6)$ & \\
\hline$\leq 1 /$ wk & $35.8(1.6)$ & $31.0(6.3)$ & \\
\hline$\geq 2 / w k$ & $40.4(1.5)$ & $41.1(5.7)$ & \\
\hline Exercise & & & 0.006 \\
\hline None & $71.3(1.6)$ & $85.7(3.4)$ & \\
\hline$\leq 2 / w k$ & $14.8(1.2)$ & $7.5(2.8)$ & \\
\hline$\geq 3 / w k$ & $13.9(1.2)$ & $6.7(2.2)$ & \\
\hline
\end{tabular}

Values are presented as mean \pm standard error or estimated \% (standard error). All data are weighted to the residential population of Korea. Calculated by complex sample general linear model and complex sample logistic regression analysis. ASM, appendicular skeletal muscle.

*Unweighted sample size.
$(\mathrm{P}=0.618)$.

The proportion of coffee consumption by stratified frequencies was significantly different between the two groups $(\mathrm{P}=0.034)$. Additionally, the ratio of the number of persons who drank less than 1 cup of coffee per day and 1 cup per day was relatively higher in the group with sarcopenia than in the group without sarcopenia ( $43.4 \%$ and $28.2 \%$ in the group with sarcopenia versus $30.7 \%$ and $27.1 \%$ in the group without sarcopenia, respectively). Furthermore, the ratios were relatively lower as daily coffee consumption increased to 2 cups and 3 or more cups (18.8\% and $9.6 \%$ in the group with sarcopenia versus $24.4 \%$ and $17.9 \%$ in the group without sarcopenia).

\section{Baseline Characteristics of the Subjects According to Coffee Consumption Frequency}

In the unweighted KNHANES data, there were 583 men who consumed less than 1 cup of coffee per day, 476 who drank 1 cup per day, 423 who drank 2 cups per day, and 299 who consumed 3 or more cups. The mean ages were lower as the daily coffee consumption amount increased $(\mathrm{P}=0.001)$, but there were no differences in mean height, weight, waist circumference, body mass index, amount of protein intake, or exercise frequency by coffee consumption. Additionally, we observed no significant differences between the means of ASM and SMI by each coffee consumption group $(\mathrm{P}=0.113, \mathrm{P}=0.243$, respectively). Among the factors we included in the regression model, smoking, alcohol consumption rate, household income, education, and total energy intake amount were significantly different by daily coffee consumption (Table 2).

\section{Relationship between Coffee Consumption and Sarcopenia}

Compared to the reference group of individuals whose daily coffee consumption was $<1$ cup, sarcopenia was significantly lower in people whose daily consumption was at least 3 cups (odds ratio [OR], 0.38; $95 \%$ confidence interval [CI], 0.18 to 0.80 ), while the prevalence of sarcopenia was not significantly lower for persons who consumed 1 or 2 cups of coffee a day ( 1 cup: OR, 0.74; 95\% CI, 0.43 to 1.26; 2 cups: OR, $0.54 ; 95 \%$ CI, 0.29 to 1.03 ) without any adjustment (model 1 in Table 3 ). This result was also observed after adjusting for covariates as shown in Table 3. Notably, the prevalence of sarcopenia was significantly lower in individuals who drank at least 3 cups of coffee per day, even after adjusting for age, smoking, alcohol, exercise, education, household income, occupational status, protein intake, and energy intake (adjusted OR, 0.44; 95\% CI, 0.21 to 0.94) (model 5 in Table 3).

The prevalence of sarcopenia was $7 \%(n=125)$ in the unweighted study population, while the estimated rate of sarcopenia was $9.0 \%$ among people who drank less than one cup of coffee per day, $6.8 \%$ among individuals who drank 1 cup per day, $5.1 \%$ in persons who consumed 2 cups of coffee per day, and 3.6\% among individuals who drank at least 3 cups of coffee per day, as shown in Figure 1 (P for trend=0.007). After adjustment for age (model 2), the estimated prevalence of sarcopenia still decreased significantly as daily coffee con- 
Table 2. Anthropometric and demographic characteristics according to coffee consumption $\left(\mathrm{N}=1,781^{*}\right)$

\begin{tabular}{|c|c|c|c|c|c|}
\hline \multirow{2}{*}{ Characteristic } & \multicolumn{4}{|c|}{ Coffee consumption (cup/d) } & \multirow{2}{*}{ P-value } \\
\hline & $<1\left(\mathrm{~N}=583^{\star}\right)$ & $1\left(N=476^{*}\right)$ & $2\left(N=423^{\star}\right)$ & $\geq 3\left(\mathrm{~N}=299^{*}\right)$ & \\
\hline Age (y) & $68.9 \pm 0.33$ & $68.5 \pm 0.38$ & $67.9 \pm 0.33$ & $66.8 \pm 0.42$ & 0.001 \\
\hline $60-69$ & $57.5(2.3)$ & $60.2(2.8)$ & $64.6(2.5)$ & $71.1(3.0)$ & 0.032 \\
\hline $70-79$ & $35.9(2.2)$ & $33.8(2.6)$ & $31.0(2.3)$ & $25.9(2.9)$ & \\
\hline$\geq 80$ & $6.6(1.2)$ & $6.0(1.4)$ & $4.4(1.1)$ & $3.1(1.2)$ & \\
\hline Height (cm) & $165.3 \pm 0.31$ & $165.6 \pm 0.29$ & $166.0 \pm 0.33$ & $166.2 \pm 3.36$ & 0.234 \\
\hline Weight (kg) & $63.7 \pm 0.54$ & $64.6 \pm 0.51$ & $65.2 \pm 0.59$ & $65.2 \pm 0.66$ & 0.225 \\
\hline Waist circumference (cm) & $85.2 \pm 0.48$ & $85.4 \pm 0.48$ & $85.8 \pm 0.57$ & $85.5 \pm 0.65$ & 0.854 \\
\hline Body mass index $\left(\mathrm{kg} / \mathrm{m}^{2}\right)$ & $23.2 \pm 0.16$ & $23.5 \pm 0.16$ & $23.6 \pm 0.19$ & $23.6 \pm 0.23$ & 0.378 \\
\hline ASM mass (kg) & $19.8 \pm 0.19$ & $20.1 \pm 0.15$ & $20.2 \pm 0.17$ & $20.5 \pm 0.18$ & 0.113 \\
\hline ASM/height ${ }^{2}\left(\mathrm{~kg} / \mathrm{m}^{2}\right)$ & $7.2 \pm 0.06$ & $7.3 \pm 0.04$ & $7.3 \pm 0.05$ & $7.4 \pm 0.06$ & 0.243 \\
\hline Hypertension & $37.7(2.6)$ & $40.5(2.7)$ & $40.1(2.9)$ & $38.9(3.8)$ & 0.888 \\
\hline Diabetes mellitus & $15.4(2.1)$ & $14.5(1.9)$ & $16.7(2.1)$ & $15.8(2.6)$ & 0.894 \\
\hline Dyslipidemia & $6.4(1.3)$ & $5.9(1.4)$ & $5.3(1.2)$ & $6.7(2.0)$ & 0.908 \\
\hline Osteoarthritis & $12.6(2.0)$ & $10.1(1.9)$ & $11.2(2.2)$ & $9.0(1.9)$ & 0.621 \\
\hline Smoking & & & & & 0.001 \\
\hline Never & $21.3(2.0)$ & $14.7(1.9)$ & $13.3(2.1)$ & $10.5(2.2)$ & \\
\hline Former & $25.6(2.2)$ & $26.5(2.4)$ & $32.9(3)$ & $22.7(3.1)$ & \\
\hline Current & $53.0(2.6)$ & $58.7(2.7)$ & $53.8(3.3)$ & $66.8(3.2)$ & \\
\hline Alcohol & & & & & 0.039 \\
\hline Never & $22.7(2.1)$ & $22.9(2.4)$ & $20.8(2.3)$ & $32.6(3.4)$ & \\
\hline$\leq 1 /$ wk & $34.9(2.7)$ & $37.1(2.9)$ & $34.5(2.8)$ & $35.5(3.6)$ & \\
\hline$\geq 2 / w k$ & $42.4(2.8)$ & $40.0(2.8)$ & $44.7(2.7)$ & $31.9(3.3)$ & \\
\hline Exercise & & & & & 0.790 \\
\hline None & $74.6(2.3)$ & $71.7(2.9)$ & $73.0(2.9)$ & $68.0(3.5)$ & \\
\hline$\leq 2 / w k$ & $12.5(1.6)$ & $15.1(2.4)$ & $13.4(2.2)$ & $17.3(2.8)$ & \\
\hline$\geq 3 / w k$ & $12.9(1.9)$ & $13.2(1.9)$ & $13.6(2.5)$ & $14.7(2.8)$ & \\
\hline Household income & & & & & $<0.001$ \\
\hline Low & $45.4(2.7)$ & $40.8(2.6)$ & $28.8(2.7)$ & $29.7(3.4)$ & \\
\hline Low-moderate & $28.5(2.5)$ & $27.5(2.2)$ & $31.3(2.8)$ & $27.1(3.4)$ & \\
\hline Moderate-high & $13.8(1.8)$ & $17.9(2.1)$ & $21.0(2.3)$ & $25.9(2.9)$ & \\
\hline High & $12.3(2.1)$ & $13.8(2.0)$ & $18.9(2.4)$ & $17.3(2.9)$ & \\
\hline Education (y) & & & & & 0.002 \\
\hline$\leq 6$ & $51.5(2.7)$ & $40.0(2.8)$ & $41.5(3.0)$ & $41.8(3.8)$ & \\
\hline $7-9$ & $20.8(2.2)$ & $20.6(2.3)$ & $21.4(2.6)$ & $22.9(3.0)$ & \\
\hline $10-12$ & $19.6(2.1)$ & $23.8(2.2)$ & $19.9(2.4)$ & $26.8(3.1)$ & \\
\hline$\geq 13$ & $8.1(1.4)$ & $15.6(2.0)$ & $17.2(2.8)$ & $8.6(2.0)$ & \\
\hline Occupation & $55.9(2.7)$ & $51.1(3.0)$ & $55.4(3.1)$ & $64.7(3.8)$ & 0.029 \\
\hline Protein intake $(\mathrm{g} / \mathrm{d})^{\dagger}$ & $65.9 \pm 1.47$ & $67.9 \pm 1.99$ & $71.2 \pm 1.97$ & $72.1 \pm 2.34$ & 0.062 \\
\hline Total energy intake $(\mathrm{kcal} / \mathrm{d})^{\dagger}$ & $1,957 \pm 31.5$ & $1,994 \pm 40.1$ & $2,101 \pm 44.8$ & $2,123 \pm 50.2$ & 0.006 \\
\hline
\end{tabular}

Values are presented as mean \pm standard error or estimated \% (standard error). All data are weighted to the residential population of Korea. Calculated by complex sample general linear model and complex sample logistic regression analysis.

ASM, appendicular skeletal muscle.

*Unweighted sample size. 'Daily energy and protein intakes were assessed using the 24-hour recall method of the nutrition survey of the Korea National Health and Nutrition Examination Survey.

sumption increased ( $\mathrm{P}$ for trend=0.025). This significant pattern continued in the multivariate-adjusted model 3,4 , and 5 as shown in Table 3 (P for trends $=0.011,0.020$, and 0.026, respectively).

\section{DISCUSSION}

Because sarcopenia is mainly related to aging, we expected the mean age of the group of sarcopenia to be higher than the group without sarcopenia, and the analyzed results were in good accord with the ex- pected results (72.0 years old in persons with sarcopenia versus 67.9 years old in individuals without sarcopenia). In the present study, coffee consumption was associated with a significantly decreased risk of sarcopenia. This observed association was independent of age, lifestyle (alcohol consumption, smoking, and exercise), degrees of education and household income, and nutrition status (protein and energy intake). Furthermore, a significant linear trend was observed across the levels of coffee consumption. Study participants who consumed 3 or more cups of coffee per day had a $62 \%$ lower risk of sarcopenia, 
Table 3. ORs and $95 \%$ Cls of sarcopenia by coffee consumption

\begin{tabular}{|c|c|c|c|c|c|c|c|}
\hline \multirow{3}{*}{ Model } & \multicolumn{6}{|c|}{ Coffee consumption (cup/d) } & \multirow{3}{*}{ P for trend } \\
\hline & \multicolumn{2}{|c|}{1} & \multicolumn{2}{|c|}{2} & \multicolumn{2}{|c|}{$\geq 3$} & \\
\hline & $\mathrm{OR}(\mathrm{Cl})$ & P-value & $\mathrm{OR}(\mathrm{Cl})$ & P-value & $\mathrm{OR}(\mathrm{Cl})$ & P-value & \\
\hline 1 & $0.74(0.43-1.26)$ & 0.36 & $0.54(0.29-1.03)$ & 0.07 & $0.38(0.18-0.80)$ & 0.01 & 0.007 \\
\hline 2 & $0.75(0.44-1.28)$ & 0.53 & $0.59(0.31-1.11)$ & 0.18 & $0.46(0.22-0.96)$ & 0.04 & 0.025 \\
\hline 3 & $0.69(0.39-1.22)$ & 0.44 & $0.55(0.29-1.05)$ & 0.17 & $0.41(0.19-0.85)$ & 0.02 & 0.011 \\
\hline 4 & $0.70(0.39-1.24)$ & 0.50 & $0.57(0.31-1.07)$ & 0.23 & $0.44(0.21-0.93)$ & 0.03 & 0.020 \\
\hline 5 & $0.69(0.39-1.24)$ & 0.44 & $0.60(0.32-1.12)$ & 0.25 & $0.44(0.21-0.94)$ & 0.04 & 0.026 \\
\hline
\end{tabular}

Values are presented as prevalence $\mathrm{OR}(95 \% \mathrm{Cl})$ compared with the group of coffee consumption less than 1 cup per day in each model. Model 1: not adjusted; model 2: univariate adjusted for age; model 3: multivariate adjusted for age, smoking, alcohol, and exercise; model 4: multivariate adjusted for education, household income, and occupation status in addition to the covariates included in the model 3; model 5: multivariate adjusted for protein intake and energy intake in addition to the covariates included in the model 4.

$\mathrm{OR}$, odds ratio; $\mathrm{Cl}$, confidence interval.

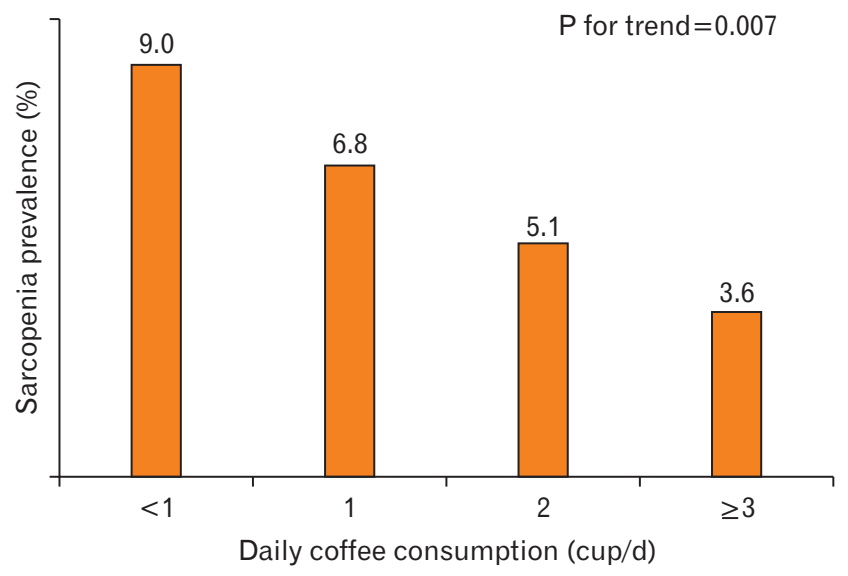

Figure 1. Unadjusted prevalence of sarcopenia according to daily coffee consumption. Sarcopenia prevalence (\%): estimated value.

compared with those who drank less than 1 cup of coffee per day in unadjusted analyses. In contrast, individuals who consumed at least 3 per day had a 57\% lower risk of sarcopenia after adjustment for the covariates mentioned above.

There are some supporting explanations about the preventive effect of coffee on sarcopenia. According to the mitochondrial free radical theory of aging, mitochondrial dysfunction due to oxidative damage to mitochondrial DNA is a major mechanism in the aging process. ${ }^{19)} \mathrm{Au}-$ tophagy is essential for proper renewal of mitochondria and maintenance of muscle mass. As a result, the autophagy rate should be increased by various stresses on muscle cells like exercise or starvation. ${ }^{20)}$ It has also been shown that autophagy is deficient in aged muscle and may affect to mitochondrial dysfunction, enhanced oxidative stress, and the decline of muscle homeostasis which occurs during the aging process. ${ }^{16)}$ According to Pietrocola et al., ${ }^{4)}$ chronic consumption of coffee diluted in the drinking water of female mice stimulated autophagy in the liver, heart, and skeletal muscle in a dose-dependent manner. Additionally, polyphenols, the main antioxidant component of coffee, have been shown to induce autophagy. ${ }^{21)}$ Because of its antioxidant properties, it is conceivable that coffee may lower the risk of sarcopenia by reducing oxidative stress of mitochondria of muscle cells.
For the definition of sarcopenia, SMI could be calculated in 2 methods representatively; ASM divided by height squared (ASM/height ${ }^{2}$ ) or by weight (ASM/weight). ${ }^{11,22)}$ In addition to various definition of SMI, the cutoff values could be chosen differently: (1) less than 1 SD below the mean values of healthy adults, (2) less than 2 SDs below the mean values of healthy adults, or (3) the lowest quintile of study populations. ${ }^{23,24)}$ In our study, the overall prevalence of sarcopenia was $7.5 \%$ in Korean men aged 60 years or older. We used ASM/height ${ }^{2}$ index with 2 SDs below the mean values of healthy adults as the cutoff value. There have been many investigations about sarcopenia in Korea and the prevalence of sarcopenia and its effects on various health conditions are different according to the methods used to define sarcopenia. ${ }^{25)}$ In an observational study by Kim et al. ${ }^{26)}$ on the association of sarcopenia with cardiometabolic risk factors in elderly aged 65 years or older, the prevalence of sarcopenia was $35.5 \%$ in men and $13.4 \%$ in women using the ASM/height ${ }^{2}$ index. In contrast, when they used ASM/weight index, the prevalence estimates rose to $37.3 \%$ for men and $62.6 \%$ for women. In another study, sarcopenia was defined as an ASM-adjusted weight index less than 2 SDs below the mean of healthy adults (20-39 years), and the prevalence of sarcopenia in participants older than 50 years was $7.8 \% .{ }^{27)}$ The difference of sarcopenia prevalence was due to different cutoff values of SMI and the difference in age and sex in the study population.

Several published studies offer insight into the role of dietary factors, including coffee, to prevent sarcopenia. According to Kim et al., ${ }^{28)}$ Korean men at least 65 years of age who consumed the highest quintile of vegetables, fruits and vegetables, and fruits consumption had $52 \%$, $70 \%$, and $68 \%$ lower risk of sarcopenia compared with those in the lowest quintile of vegetable, fruit, and vegetables and fruit consumption. These results were hypothesized to be related to the antioxidant effects of vegetables and fruits on sarcopenia. Kim et al. ${ }^{27)}$ also demonstrated that vitamin D levels were significantly lower in subjects with sarcopenia compared with those without sarcopenia.

In this study, we hypothesized that coffee might be associated with a reduced risk of sarcopenia in humans through its antioxidant, anti-inflammatory, and pro-autophagy effects described in previous animal trials. ${ }^{21)}$ Our results support this hypothesis. To our knowledge, this is 
the first human study to examine the correlation between coffee consumption and sarcopenia. These data support the potential role of coffee in helping prevent sarcopenia ${ }^{18)}$ via the theoretical mechanism of coffee on sarcopenia. ${ }^{29)}$ Another strong point of this study is that the study sample was obtained from a reliable nationally representative population database, lending credence to the generalizability of the findings, which were statistically significant even after adjustment for covariates including demographic and health-related variables.

This study has some limitations. First, coffee consumption was estimated by asking individuals to recall the amounts of coffee they drank daily on average over the preceding year. The data did not take into account any changes of consumption over the person's lifetime. Second, because the study was performed using a cross-sectional design, there is limitation to detect the causal relations or mechanism of action. Third, we cannot completely exclude the possibility that confounding due to consumption of herb tea, green tea, vegetables, fruits, or other substances that provide antioxidants.

In conclusion, this study is a large population-based study to examine the association between coffee consumption and sarcopenia. High consumption of coffee was associated with a lower risk of sarcopenia in elderly Korean men.

\section{CONFLICT OF INTEREST}

No potential conflict of interest relevant to this article was reported.

\section{ACKNOWLEDGMENTS}

This work was supported by a 2015 research grant from Jeju National University Hospital.

\section{REFERENCES}

1. Clifford MN. Chlorogenic acids and other cinnamates-nature, occurrence, dietary burden, absorption and metabolism. J Sci Food Agric 2000;80:1033-43.

2. Andersen LF, Jacobs DR Jr, Carlsen MH, Blomhoff R. Consumption of coffee is associated with reduced risk of death attributed to inflammatory and cardiovascular diseases in the Iowa Women's Health Study. Am J Clin Nutr 2006;83:1039-46.

3. Lopez-Garcia E, van Dam RM, Qi L, Hu FB. Coffee consumption and markers of inflammation and endothelial dysfunction in healthy and diabetic women. Am J Clin Nutr 2006;84:888-93.

4. Pietrocola F, Malik SA, Marino G, Vacchelli E, Senovilla L, Chaba K, et al. Coffee induces autophagy in vivo. Cell Cycle 2014;13:1987-94.

5. Jiang X, Zhang D, Jiang W. Coffee and caffeine intake and incidence of type 2 diabetes mellitus: a meta-analysis of prospective studies. Eur J Nutr 2014;53:25-38.

6. Pham NM, Nanri A, Kochi T, Kuwahara K, Tsuruoka H, Kurotani K, et al. Coffee and green tea consumption is associated with insulin resistance in Japanese adults. Metabolism 2014;63:400-8.

7. Larsson SC, Virtamo J, Wolk A. Coffee consumption and risk of stroke in women. Stroke 2011;42:908-12.

8. Ross GW, Abbott RD, Petrovitch H, Morens DM, Grandinetti A, Tung $\mathrm{KH}$, et al. Association of coffee and caffeine intake with the risk of Parkinson disease. JAMA 2000;283:2674-9.

9. Bonita JS, Mandarano M, Shuta D, Vinson J. Coffee and cardiovascular disease: in vitro, cellular, animal, and human studies. Pharmacol Res 2007;55:187-98.

10. Yu X, Bao Z, Zou J, Dong J. Coffee consumption and risk of cancers: a meta-analysis of cohort studies. BMC Cancer 2011;11:96.

11. Baumgartner RN, Koehler KM, Gallagher D, Romero L, Heymsfield SB, Ross RR, et al. Epidemiology of sarcopenia among the elderly in New Mexico. Am J Epidemiol 1998;147:755-63.

12. Iannuzzi-Sucich M, Prestwood KM, Kenny AM. Prevalence of sarcopenia and predictors of skeletal muscle mass in healthy, older men and women. J Gerontol A Biol Sci Med Sci 2002;57:M772-7.

13. Landi F, Cruz-Jentoft AJ, Liperoti R, Russo A, Giovannini S, Tosato M, et al. Sarcopenia and mortality risk in frail older persons aged 80 years and older: results from ilSIRENTE study. Age Ageing 2013;42:203-9.

14. Cruz-Jentoft AJ, Baeyens JP, Bauer JM, Boirie Y, Cederholm T, Landi F, et al. Sarcopenia: European consensus on definition and diagnosis: report of the European Working Group on Sarcopenia in Older People. Age Ageing 2010;39:412-23.

15. Argiles JM, Busquets S, Stemmler B, Lopez-Soriano FJ. Cachexia and sarcopenia: mechanisms and potential targets for intervention. Curr Opin Pharmacol 2015;22:100-6.

16. Marzetti E, Calvani R, Cesari M, Buford TW, Lorenzi M, Behnke BJ, et al. Mitochondrial dysfunction and sarcopenia of aging: from signaling pathways to clinical trials. Int J Biochem Cell Biol 2013;45:2288-301.

17. Hiona A, Leeuwenburgh C. The role of mitochondrial DNA mutations in aging and sarcopenia: implications for the mitochondrial vicious cycle theory of aging. Exp Gerontol 2008;43:24-33.

18. Guo Y, Niu K, Okazaki T, Wu H, Yoshikawa T, Ohrui T, et al. Coffee treatment prevents the progression of sarcopenia in aged mice in vivo and in vitro. Exp Gerontol 2014;50:1-8.

19. Miquel J, Economos AC, Fleming J, Johnson JE Jr. Mitochondrial role in cell aging. Exp Gerontol 1980;15:575-91.

20. Mizushima N, Yamamoto A, Matsui M, Yoshimori T, Ohsumi Y. In vivo analysis of autophagy in response to nutrient starvation using transgenic mice expressing a fluorescent autophagosome marker. Mol Biol Cell 2004;15:1101-11.

21. Pallauf K, Rimbach G. Autophagy, polyphenols and healthy ageing. Ageing Res Rev 2013;12:237-52.

22. Janssen I, Heymsfield SB, Ross R. Low relative skeletal muscle mass (sarcopenia) in older persons is associated with functional impairment and physical disability. J Am Geriatr Soc 2002;50:889-96.

23. Di Monaco M, Castiglioni C, De Toma E, Gardin L, Giordano S, Di Monaco R, et al. Presarcopenia and sarcopenia in hip-fracture women: prevalence and association with ability to function in activities of daily living. Aging Clin Exp Res 2015;27:465-72.

24. Lee WJ, Liu LK, Peng LN, Lin MH, Chen LK; ILAS Research Group. Comparisons of sarcopenia defined by IWGS and EWGSOP criteria among older people: results from the I-Lan longitudinal aging study. J Am Med Dir Assoc 2013;14:528.e1-7.

25. Kim KM, Lim S, Choi KM, Kim JH, Yu SH, Kim TN, et al. Sarcopenia in Korea: prevalence and clinical aspects. J Korean Geriatr Soc 2015;19:1-8. 
26. Kim JH, Hwang Bo Y, Hong ES, Ohn JH, Kim CH, Kim HW, et al. Investigation of sarcopenia and its association with cardiometabolic risk factors in elderly subjects. J Korean Geriatr Soc 2010;14:121-30.

27. Kim MK, Baek KH, Song KH, Kang MI, Park CY, Lee WY, et al. Vitamin D deficiency is associated with sarcopenia in older Koreans, regardless of obesity: the Fourth Korea National Health and Nutrition Examination Surveys (KNHANES IV) 2009. J Clin Endocrinol Metab 2011;96:
3250-6.

28. Kim J, Lee Y, Kye S, Chung YS, Kim KM. Association of vegetables and fruits consumption with sarcopenia in older adults: the Fourth Korea National Health and Nutrition Examination Survey. Age Ageing 2015;44:96-102.

29. Dirks-Naylor AJ. The benefits of coffee on skeletal muscle. Life Sci 2015;143:182-6. 